\title{
Revealing microstructure and dislocation behavior in BAIN/AIGaN heterostructures
}

\author{
Haiding Sun ${ }^{1}$, Feng Wu${ }^{1}$, Young Jae Park², T. M. Al tahtamouni ${ }^{3}$, Che-Hao Liao ${ }^{1}$, Wenzhe Guo ${ }^{1}$, Nasir Alfaraj ${ }^{1}$, \\ Kuang-Hui Li ${ }^{1}$, Dalaver H. Anjum ${ }^{4}$, Theeradetch Detchprohm², Russell D. Dupuis², and Xiaohang Li ${ }^{1}$ \\ ${ }^{1}$ King Abdullah University of Science and Technology (KAUST), Advanced Semiconductor Laboratory, Thuwal 23955-6900, Saudi Arabia \\ ${ }^{2}$ Center for Compound Semiconductors and School of Electrical and Computer Engineering, Georgia Institute of Technology, Atlanta, GA 30332, U.S.A. \\ ${ }^{3}$ Materials Science and Technology Program, College of Arts and Sciences, Qatar University, Doha 2713, Qatar \\ ${ }^{4}$ King Abdullah University of Science and Technology (KAUST), Imaging and Characterization Core Laboratory, Thuwal 23955-6900, Saudi Arabia \\ Received September 23, 2017; accepted November 28, 2017; published online December 18, 2017
}

We reveal the microstructure and dislocation behavior in 20-pair $\mathrm{B}_{0.14} \mathrm{Al}_{0.86} \mathrm{~N} / \mathrm{Al}_{0.70} \mathrm{Ga}_{0.30} \mathrm{~N}$ multiple-stack heterostructures (MSHs) exhibiting an increasing dislocation density along the $c$-axis, which is attributed to the continuous generation of dislocations (edge and mixed-type) within the individual $\mathrm{B}_{0.14} \mathrm{Al}_{0.86} \mathrm{~N}$ layers. At the $\mathrm{MSH}$ interfaces, the threading dislocations were accompanied by a string of $\mathrm{V}$-shape pits extending to the surface, leading to interface roughening and the formation of surface columnar features. Strain maps indicated an approximately $1.5 \%$ tensile strain and $1 \%$ compressive strain in the $\mathrm{B}_{0.14} \mathrm{Al}_{0.86} \mathrm{~N}$ and $\mathrm{Al}_{0.70} \mathrm{Ga}_{0.30} \mathrm{~N}$ layers, respectively. Twin structures were observed, and the $\mathrm{MSH}$ eventually changed from monocrystalline to polycrystalline. (C) 2018 The Japan Society of Applied Physics

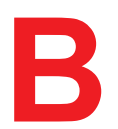

oron-aluminum nitride (BAlN) is an emerging IIInitride alloy that has a large bandgap comparable to that of AlN and Al-rich AlGaN. ${ }^{1)}$ It is promising for deep-ultraviolet (DUV) distributed Bragg reflectors (DBRs), and thus surface-emitting lasers. ${ }^{2)}$ This is because of the considerable reduction in the refractive index with the increase of the $\mathrm{B}$ content with respect to AlN. ${ }^{3)}$ Our recent study of the band alignment of the BAlN/AlGaN heterojunction shows its potential for the electron-blocking layers of optoelectronics and barriers of power-electronic devices due to the unique band offset with conventional InAlGaN alloys. ${ }^{4)}$ Despite the potential of BAIN, the scope of experimental studies has been limited, especially in terms of the growth of single-phase wurtzite BAIN layers with a high B content and large thickness. Previous reports on BAlN alloy growth indicate a B content of merely $1-2 \%$ in the layers owing to phase separation, limited B diffusion, and parasitic reactions. ${ }^{5)} \mathrm{Li}$ et al. demonstrated a far higher B content of $12 \% .^{6,7)}$ However, the singlephase wurtzite structure was only observed in the first $10 \mathrm{~nm}$ or a thinner region of the BAlN layers. Above this thickness, a polycrystalline phase was formed, which is undesirable in wurtzite device structures. Recently, we demonstrated a significant increase in the thickness (i.e., $100 \mathrm{~nm}$ ) and B content to $14.4 \%$ for single-phase wurtzite BAlN layers. ${ }^{8,9)}$ This result provides the foundation for the development of BAlN-based heterostructures.

Abid and $\mathrm{Li}$ et al. performed growth and characterization studies on BAIN/AlN heterostructures. ${ }^{3,7)}$ However, these studies were constrained by the small thickness of the wurtzite phase. Additionally, the application of the BAIN/ AIN heterostructures could be limited because of the similar properties, such as the bandgap, band offset, and refractive index, even for the non-active device layer. Moreover, we found that the bandgap of BAIN transitioned from direct to indirect as the B content increased to $12 \% .{ }^{1)}$ This result indicates that it is unlikely for BAlN to be the sole material for the active layer of future high-performance DUV devices. Therefore, BAlN would probably have to be integrated with Al-rich AlGaN to form heterostructures for application in DUV devices. Thus far, there have been limited experimental reports concerning the structural characterization of BAIN/ AlGaN heterostructures with a high $\mathrm{B}$ content.

In this study, we grew BAlN/AlGaN multiple-stack heterostructures (MSHs) using metalorganic chemical vapor deposition (MOCVD). We conducted detailed characterizations related to the microstructure, defect formation, and strain within the BAlN and AlGaN layers. Our experimental analysis provides insights for future research and development of the BAlN/AlGaN heterostructures by improving the quality of such junctions.

The MSHs used in this study comprised 20 pairs of 35-nm $\mathrm{B}_{0.14} \mathrm{Al}_{0.86} \mathrm{~N} / 30 \mathrm{~nm} \mathrm{Al} \mathrm{Al}_{0.7} \mathrm{Ga}_{0.3} \mathrm{~N}$ and were grown on a $3-\mu \mathrm{m}-$ thick AlN template layer on a $c$-plane sapphire substrate, ${ }^{10,11)}$ with the first and last $\mathrm{MSH}$ layers being $\mathrm{B}_{0.14} \mathrm{Al}_{0.86} \mathrm{~N}$ and $\mathrm{Al}_{0.7} \mathrm{Ga}_{0.3} \mathrm{~N}$, respectively. The AlN templates had a roughness of $\sim 0.5 \mathrm{~nm}$, with an estimated threading dislocation (TD) density of approximately $10^{9} / \mathrm{cm}^{2}$. Details regarding the AlN templates can be found in Refs. 10 and 11. The MSH layer thicknesses were chosen to match those of a $\mathrm{B}_{0.14} \mathrm{Al}_{0.86} \mathrm{~N} /$ $\mathrm{Al}_{0.7} \mathrm{Ga}_{0.3} \mathrm{~N}$ DBR having peak reflectivity at approximately $\sim 300 \mathrm{~nm}$. The $\mathrm{B}_{0.14} \mathrm{Al}_{0.86} \mathrm{~N}$ and $\mathrm{Al}_{0.7} \mathrm{Ga}_{0.3} \mathrm{~N}$ layers had refractive indices of approximately $2.1^{3)}$ and $2.3,{ }^{12)}$ respectively, at $300 \mathrm{~nm}$. The calculated theoretical peak reflectivity closes to be $95 \%$ with a stopband of $20 \mathrm{~nm}$ (not shown). Triethylboron, trimethylaluminium, trimethylgallium, and $\mathrm{NH}_{3}$ were used as precursors, with the carrier gas being $\mathrm{H}_{2}$. The detailed growth conditions of the $\mathrm{B}_{0.14} \mathrm{Al}_{0.86} \mathrm{~N}$ layers were similar to those we previously reported. ${ }^{8)}$ The growth temperature was maintained at $910^{\circ} \mathrm{C}$.

Subsequent to the growth, we examined the surface morphology of the MSH using atomic force microscopy (AFM). As shown in the inset of Fig. 1, the MSH surface was dominated by columnar structures with horizontal diameters between 100 and $200 \mathrm{~nm}$. The root-mean-square roughness was $6.9 \mathrm{~nm}$. The formation of the columnar structures was likely caused by the BAIN layers, as previous studies showed that the BAIN layers had a similar surface morphology. However, its formation mechanism was not well understood. ${ }^{7,8)}$ To elucidate this, high-angle annular dark-field (HAADF) scanning transmission electron microscopy (STEM) experiments were performed using an FEI probe-corrected Titan microscope operated at an acceleration voltage of $300 \mathrm{kV}$. High-resolution transmission electron microscopy (HR-TEM) specimens were prepared by employing an FEI Helios dualbeam focused ion beam scanning electron microscope system with a $\mathrm{Ga}$ ion source.

Figure 1 presents a cross-sectional HAADF-STEM image of the MSH. The bright and dark layers correspond to the 


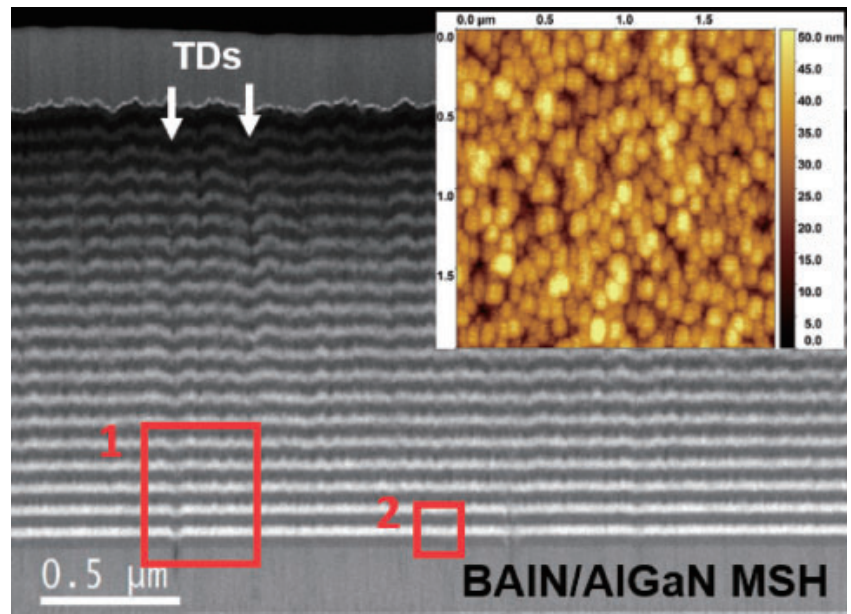

Fig. 1. A cross-sectional HAADF-STEM image of the MSH where the red boxes labeled as "1" and "2" are used in the discussion of Figs. 2 and 3, respectively. Inset shows an AFM image.

$\mathrm{AlGaN}$ and BAlN layers, respectively. All 20 pairs were present. The MSH interface was relatively smooth for the first few pairs and became rougher along the growth direction. Eventually, this led to the columnar surface morphology shown in Fig. 1. During the gradual roughening, the density of the TDs increased, and V-shape pits (V-pits) were consequently formed along the $c$-axis at the MSH interface, as shown in Fig. 1. Most of the TDs were generated within the MSH, and a few originated from the AlN template. On average, the distance between two V-pit centers on the MSH surface was in the range of 100 to $200 \mathrm{~nm}$, which is consistent with the diameter of the columnar structures from Fig. 1. This indicates that the columnar growth mode was caused by the V-pit formation associated with the TD.

To investigate the V-pit formation, higher-magnification HAADF-STEM experiments were conducted in the area enclosed by the red box (area "1") in Fig. 1, including a V-pit as well as part of the AIN template and the MSH. A TD originated from the AlN template and propagated upwards along the $c$-axis, as shown in Fig. 2(a). When the TD reached the first BAIN/AlGaN interface, a V-pit was formed, as highlighted by the red V-mark in Fig. 2(a). The growth of BAlN/AlGaN interfaces followed, creating a string of the V-pits extending to the surface, as schematically shown in Fig. 2(b).

For the $\mathrm{InGaN} / \mathrm{GaN}$ heterostructures, it has been reported that the (1011) facet constituted the TD-induced V-pit, which was attributed to the relaxation of the stored strain around the dislocation core. ${ }^{13-16)}$ It is noted that individual V-pits in the $\mathrm{InGaN} / \mathrm{GaN}$ heterostructures can keep enlarging with the growth and eventually form large inverted-pyramid surface pits, as shown in Fig. 2(c). The V-pits in our studies may also be created by the strain relaxation near the dislocation core because of the large lattice mismatch between the BAlN and AlGaN layers. However, it is important to note that the size evolution was apparently different. Although the V-pits slowly became larger along the $c$-axis, their sizes were still comparable to each other, as shown in Fig. 1.

The respective surface kinetics can explain the difference of the V-pit size evolution between the InGaN/GaN heterostructures and the BAIN/AlGaN MSH. For the InGaN/GaN

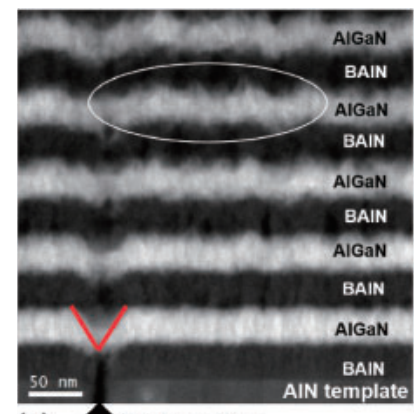

(a) Tislocation

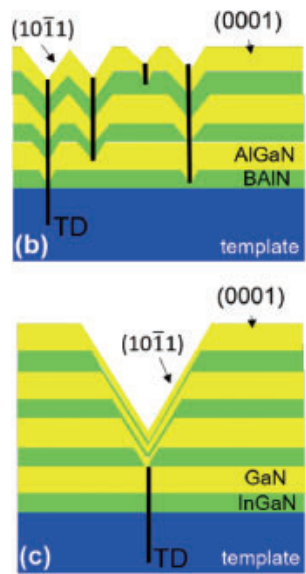

Fig. 2. (a) HAADF-STEM image of the red box "1" in Fig. 1 (b) Schematic model of the V-pit formation in (b) the MSH and (c) in an $\mathrm{InGaN} / \mathrm{GaN}$ heterostructure.
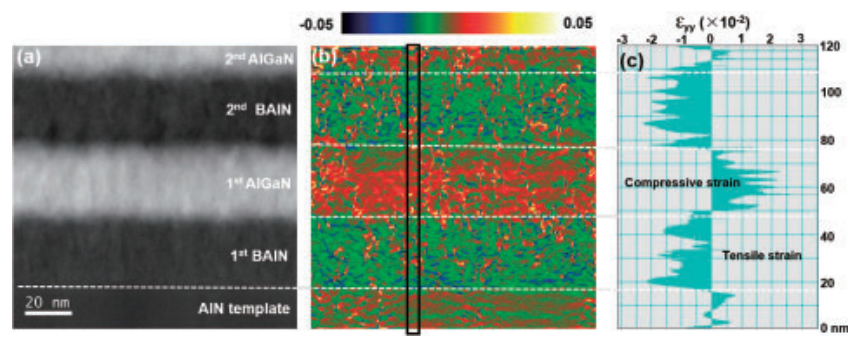

Fig. 3. HAADF-STEM image and strain mapping of the MSH. The figures include the first four layers of the MSH and an approximately 20-nm AlN template layer. (a) Image from the area of the red box labelled " 2 " in Fig. 1. (b) Corresponding strain maps obtained using the GPA for the image in (a) along the growth direction $(0002), \varepsilon_{y y}$. (c) Strain profile from the black boxed regions in (b).

heterostructures, as the (1011) plane is energetically less favorable for the arriving In and Ga adatoms than the (0001) plane, these adatoms with a large diffusion length tend to migrate to the (0001) plane, leading to a higher growth rate on the (0001) plane but a reduced growth rate on the (1011) plane. ${ }^{15,16)}$ There have been few reports regarding the surface energy of $\mathrm{B}$ and $\mathrm{Al}$ adatoms on the (0001) and (1011) planes. Regardless, any difference of the surface energy can be overcome by their smaller diffusion length, which means that the B and Al adatoms tend to stay on the (1011) and (0001) planes, thereby mitigating the higher growth rate on the (0001) plane and thus preventing the V-pit from rapidly growing. In the meantime, the $\mathrm{Ga}$ adatoms as a minor part of the precursors slightly increase the growth rate on the (0001) plane, which leads to a slowly enlarging size for the V-pit, as shown in Fig. 1 and Fig. 2(b). This possibly indicates a larger Ga composition of the AlGaN layer on the (0001) plane than on the (1011) plane, which is worthy of future study.

As most of the V-pits were associated with the TDs generated within the MSH, it was important to understand the mechanism of the TD generation. Geometric phase analysis (GPA) was conducted on the HAADF STEM image of the MSH shown in Fig. 3(a) to analyze the strain, as the lattice mismatch between the MSH layers was considerable, which might have led to dislocations due to the localized strain relaxation. ${ }^{1,17)}$ The reciprocal lattice vectors $\boldsymbol{g}=0002$ and $g=11 \overline{2} 0$ were selected from the image power spectrum to 
calculate the phase images and generate the subsequent twodimensional strain tensors during the GPA processing. ${ }^{18)}$ The strain was referenced from the AlN template and was thus relative. Figure 3(b) shows a map of the strain along the $c$-axis $\left(\varepsilon_{y y}\right)$. The one-dimensional strain profile in the black rectangular box in Fig. 3(b) is shown in Fig. 3(c). It shows approximately $-1.5 \%$ and $+1 \%$ strain within the BAIN and AlGaN layers along the $c$-axis, respectively. This means that the BAlN and AlGaN layers were under tensile and compressive strain, respectively. These measured values slightly deviate from the theoretical strain values of the BAlN and $\mathrm{AlGaN}$ layers, which are calculated to be $-2.15 \%$ and $+0.74 \%$, respectively, as estimated from the lattice constants by assuming that the $\mathrm{BAlN}^{1,19)}$ and $\mathrm{AlGaN}$ layers are fully strained to the fully relaxed AIN template. This deviation is reasonable because in the real case, the bottom AlN may not be fully relaxed; additionally, there is strain relaxation via dislocation formation in the epilayers.

In the strain map of Fig. 3(b), the first BAIN layer exhibits a large density of blue and yellowish spots with a significantly larger strain than the layer average. The strain discontinuity associated with the presence of dislocations is represented. ${ }^{20,21)}$ In the first BAlN layer, some dislocations were connected with the ones in the AlN template, indicating that they were a result of the dislocation propagation from the template, while several were generated at the BAlN/AlN interface. We also noticed that the majority of the dislocations were formed within the layer. Subsequently, as the first AlGaN layer was grown, some dislocations propagated upwards, constituting most of the dislocations therein. Furthermore, some of the dislocations in the first $\mathrm{AlGaN}$ layer propagated into the second BAIN layer. Adding the dislocations formed therein, the dislocation density of the second BAIN layer was determined to be larger than that of the first BAIN layer by counting the density of dislocation cores (blue and yellowish spots) from the strain map in Fig. 3(b). We conjecture that the dislocation density continued to rise within the subsequently grown BAIN layers, which explained the observation in Fig. 1. This observation differs from what is normally observed in AlGaN/AlGaN heterostructures, where the dislocation density may decrease along the growth direction owing to interface screening. ${ }^{22)}$ Moreover, the difference in the dislocation source between the $\mathrm{AlGaN}$ and BAlN layers indicates that the intrinsic growth challenges, such as the short diffusion length and strong parasitic reactions, ${ }^{6,8)}$ were mainly responsible for the higher dislocation density in the BAlN layers. This is because both layers were under similar strain, which, if related, generally led to misfit dislocations at the interfaces.

The types of dislocations were further studied by HR-TEM, focusing on the first BAIN layer. The first BAIN layer had a significantly higher dislocation density than the AlN template, as shown in Fig. 4(a). inverse Fourier-filtered images were obtained corresponding to the (1010) and (0002) diffraction spots, as shown in Figs. 4(b) and 4(c), respectively. According to the Burgers vectors of the dislocations in wurtzite III-nitrides, ${ }^{23)}$ the edge, screw, and mixed dislocations were identified, as indicated by red, white, and black circles, respectively. Planar defects were not present. Most of the dislocations were edge and mixed dislocations, mainly at the interface. Furthermore, the dislocations either formed a
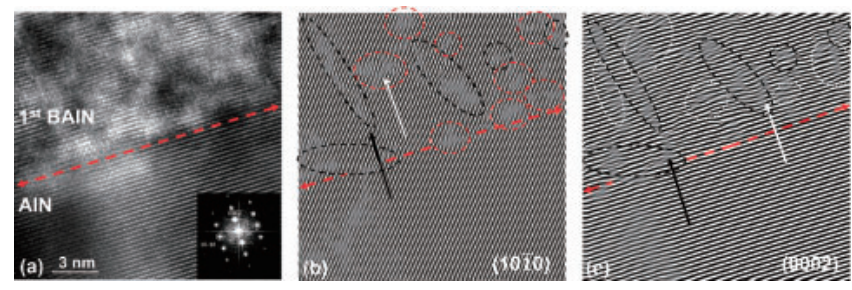

Fig. 4. (a) $[1 \overline{1} 00]$ zone-axis HR-TEM image at the BAlN/AlN interface The inset shows the corresponding FFT pattern. (b, c) Inverse Fourier-filtered images obtained using the circled spots indicated in the inset of (a) corresponding to the (1010) and (0002) planes, respectively. The red, white, and black dashed circles represent edge, screw, and mixed dislocations. The white and black arrows indicate different behaviors of dislocations.
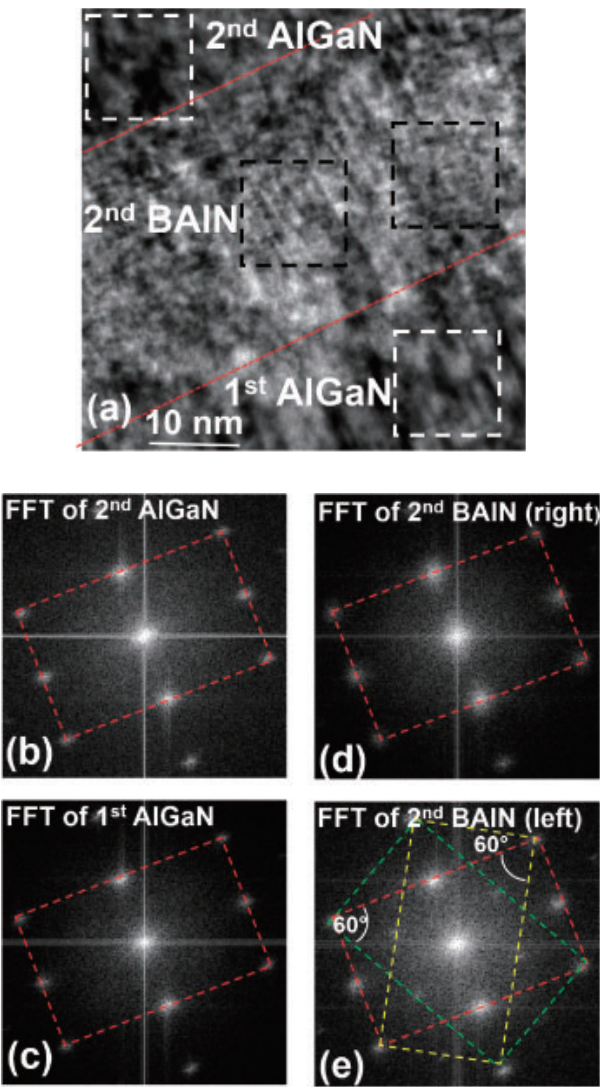

Fig. 5. (a) [1 $1 \overline{1} 00]$ zone axis HR-TEM image recorded at the $1 \mathrm{st} \mathrm{AlGaN} /$ 2nd BAlN/2nd AlGaN region. The red dashed lines indicate the interface between the layers. (b, c) FFT patterns of the regions in the white dashed boxes marked in the 2 nd AlGaN and 1 st AlGaN layer in (a). (d, e) FFT patterns of the regions in the black dashed boxes marked in the second BAlN layer in (a).

continuous path along the growth direction (black arrow) or were annihilated by forming loops (white arrow), which is consistent with the observation of Fig. 3(b).

In addition to the generation of various types of dislocations, we observed a twin formation within the BAlN layers. Figure 5 exhibits a cross-sectional TEM image recorded at the 1st AlGaN/2nd BAlN/2nd AlGaN interface. The fast Fourier transform (FFT) diffraction patterns from the region enclosed by white boxes (the AlGaN layer) show a typical wurtzite structure with the same orientation, as shown in Figs. 5(b) and 5(c). However, the FFT patterns from the BAlN layer exhibit different orientations. Figure 5(d) shows the same orientation as the AlGaN layer, whereas in Fig. 5(e), we observe extra 

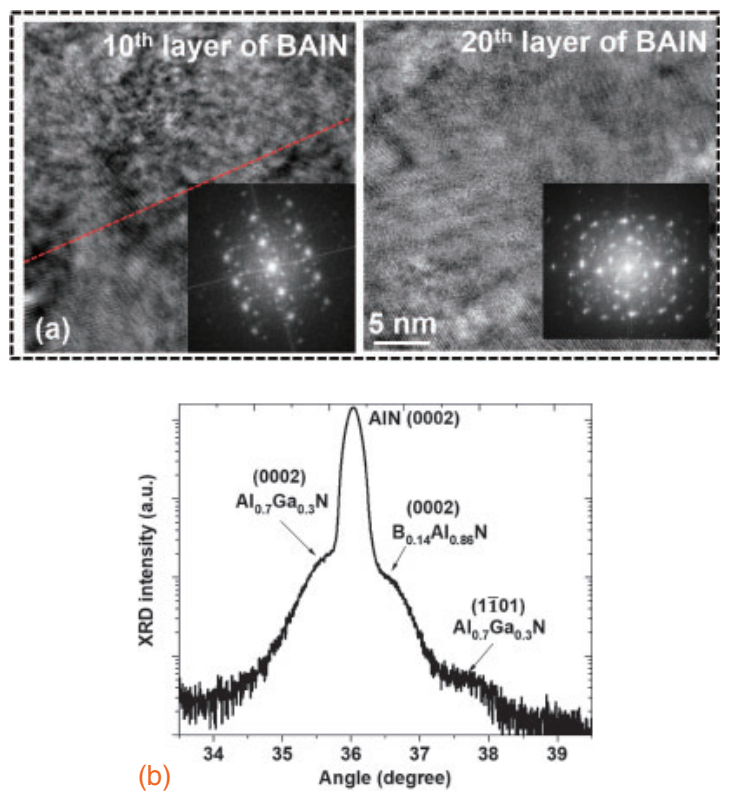

Fig. 6. (a) HR-TEM image of the 10th layer and the last BAIN layer in the MSH near the surface. The inset shows the corresponding FFT patterns. (b) $2 \theta / \omega$ XRD spectrum of the MSH.

spots in the diffraction patterns that result from a mirror reflection about certain lattice planes. It contains two twin boundaries tilted by approximately $60^{\circ}$ — one clockwise and the other counter-clockwise (marked as green and yellow lines) — with respect to the basal plane (red line), indicating the formation of the twin structures. Their mirror planes were most likely $\{1 \overline{1} 01\}$ and $\{1 \overline{1} 03\}$, as we recently reported. ${ }^{9)}$

TEM images of the 10th and the last layer (20th) of BAIN were also obtained. The region near the surface exhibited polycrystallinity, as shown in the inset of Fig. 6(a). However, according to the FFT results for the 10th layer of the BAIN in the MSH, the crystallinity was maintained relatively well along the (0002), except for the observation of the additional dots in the FFT, indicating the formation of misoriented domains in the film. An X-ray diffraction (XRD) experiment confirmed the observation. In the $2 \theta / \omega$ scan of the MSH shown in Fig. 6(b), the strongest peak related to AlN(0002) was located at $36.02^{\circ}$, which also included left and right shoulders, representing the AlGaN and BAlN (0002) peaks, respectively. A weak peak at $37.72^{\circ}$ corresponded to the wurtzite AlGaN(1101) plane, which was caused by the faceted (1101) AlGaN grains in the (0002) AlGaN matrix, indicating the polycrystallization ${ }^{5,7)}$ Fig. 6(b) shows no superlattice satellite peaks due to the MSH interfacial roughness. Because of the twin structure, V-pits, and large generation of dislocations in the BAIN layers, it was difficult to understand how the wurtzite monocrystallinity transformed into polycrystallinity, which is worthy of further study.

In summary, the microstructure of a $\mathrm{B}_{0.14} \mathrm{Al}_{0.86} \mathrm{~N} / \mathrm{Al}_{0.70^{-}}$ $\mathrm{Ga}_{0.30} \mathrm{~N}$ MSH grown via MOCVD was revealed. Within the BAIN layers, edge and mixed dislocations with a high density were formed owing to epitaxial challenges, such as the small B diffusion length and parasitic reactions. Some of the dislocations within the BAlN layers propagated upwards, leading to an increasing dislocation density along the $c$-axis, and thus a columnar surface. V-pits were formed at the MSH interface along the TDs, which led to the columnar surface. The V-pit size was relatively stable because of the similar growth rates on the (0001) and (1011) planes, which differed from the $\mathrm{V}$-pits in the $\mathrm{InGaN} / \mathrm{GaN}$ heterostructures. Twin structures were also observed in the BAlN layers. The MSH structure became polycrystalline near the surface, which is worth investigating further. The results indicate that further optimization of the BAlN growth conditions is necessary for achieving high-quality BAlN/AlGaN MSH for optical and power electronics.

Acknowledgments The KAUST authors acknowledge the support of the GCC Research Program REP/1/3189-01-01, Baseline BAS/1/1664-01-01, and Equipment BAS/1/1664-01-07. The work at QU was supported by the GCC Research Program GCC-2017-007. The work at the Georgia Institute of Technology was supported in part by DARPA under Grant No. W911NF-15-10026 and NSF under Grant No. DMR-1410874. R.D.D. acknowledges the additional support of the Steve W. Chaddick Endowed Chair in Electro-Optics and Georgia Research Alliance.

1) M. Zhang and X. Li, Phys. Status Solidi B 254, 1600749 (2017)

2) X. Li, H. Xie, J. H. Ryou, F. A. Ponce, T. Detchprohm, and R. D. Dupuis, Appl. Phys. Lett. 107, 241109 (2015).

3) M. Abid, T. Moudakir, G. Orsal, S. Gautier, A. E. Naciri, Z. Djebbour, J.-H Ryou, G. Patriarche, L. Largeau, H. J. Kim, Z. Lochner, K. Pantzas, D. Alamarguy, F. Jomard, R. D. Dupuis, J.-P. Salvestrini, P. L. Voss, and A Ougazzaden, Appl. Phys. Lett. 100, 051101 (2012).

4) H. Sun, Y. Park, K.-H. Li, C. G. Torres-Castanedo, A. Alowayed, T. Detchprohm, R. D. Dupuis, and X. Li, Appl. Phys. Lett. 111, 122106 (2017).

5) T. Akasaka and T. Makimoto, Appl. Phys. Lett. 88, 041902 (2006).

6) X. Li, S. Sundaram, Y. El Gmili, T. Moudakir, F. Genty, S. Bouchoule, G. Patriarche, R. D. Dupuis, P. L. Voss, J.-P. Salvestrini, and A. Ougazzaden, Phys. Status Solidi A 212, 745 (2015).

7) X. Li, S. Sundaram, Y. El Gmili, F. Genty, S. Bouchoule, G. Patriarche, P. Disseix, F. Réveret, J. Leymarie, J.-P. Salvestrini, R. D. Dupuis, P. L. Voss, and A. Ougazzaden, J. Cryst. Growth 414, 119 (2015).

8) X. Li, S. Wang, H. Liu, F. A. Ponce, T. Detchprohm, and R. D. Dupuis, Phys. Status Solidi B 254, 1600699 (2017)

9) S. Wang, X. Li, A. M. Fischer, T. Detchprohm, R. D. Dupuis, and F. A. Ponce, J. Cryst. Growth 475, 334 (2017).

10) H. Sun, F. Wu, T. M. Al tahtamouni, N. Alfaraj, K.-H. Li, T. Detchprohm, R. D. Dupuis, and X. Li, J. Phys. D 50, 395101 (2017).

11) H. Sun, F. Wu, Y. J. Park, T. M. Al tahtamouni, K.-H. Li, N. Alfaraj, T. Detchprohm, R. D. Dupuis, and X. Li, Appl. Phys. Lett. 110, 192106 (2017).

12) D. Brunner, H. Angerer, E. Bustarret, R. Freudenberg, R. Höpler, R. Dimitrov, O. Ambacher, and M. Stutzmann, J. Appl. Phys. 82, 5090 (1997)

13) X. H. Wu, D. Kapolnek, E. J. Tarsa, B. Heying, S. Keller, B. P. Keller, U. K. Mishra, S. P. DenBaars, and J. S. Speck, Appl. Phys. Lett. 68, 1371 (1996).

14) M. Shiojiri, C. C. Chuo, J. T. Hsu, J. R. Yang, and H. Saijo, J. Appl. Phys 99, 073505 (2006).

15) J. E. Northrup and J. Neugebauer, Phys. Rev. B 60, R8473 (1999).

16) J. E. Northrup and J. Neugebauer, Appl. Phys. Lett. 74, 2319 (1999).

17) Z. Dridi, B. Bouhafs, and P. Ruterana, Semicond. Sci. Technol. 18, 850 (2003).

18) J. L. Rouvière, E. Prestat, P. Bayle-Guillemaud, M. D. Hertog, C. Bougerol, D. Cooper, and J. Zuo, J. Phys.: Conf. Ser. 471, 012001 (2013).

19) K. Liu, H. Sun, F. AlQatari, W. Guo, X. Liu, J. Li, C. G. Torres-Castanedo, and X. Li, Appl. Phys. Lett. 111, 222106 (2017).

20) S. L. Rhode, M. K. Horton, S.-L. Sahonta, M. J. Kappers, S. J. Haigh, T. J. Pennycook, C. McAleese, C. J. Humphreys, R. O. Dusane, and M. A. Moram, J. Appl. Phys. 119, 105301 (2016).

21) M. J. Hÿtch, J. Putaux, and J. Pénisson, Nature 423, 270 (2003).

22) H. Wang, J. P. Zhang, C. Q. Chen, Q. Fareed, J. W. Yang, and A. Khan, Appl. Phys. Lett. 81, 604 (2002).

23) X. H. Wu, L. M. Brown, D. Kapolnek, S. Keller, B. Keller, S. P. DenBaars, and J. S. Speck, J. Appl. Phys. 80, 3228 (1996). 